Return to the Manage Active Submissions page at http://spie.org/app/submissions/tasks.aspx and approve or disapprove this submission. Your manuscript will not be published without this approval. Please contact author_help@spie.org with any questions or concerns.

\title{
Core-doped Nd:YAG disk solar laser uniformly pumped by six Fresnel lenses
}

\author{
D. Garcia, D. Liang* and J. Almeida \\ CEFITEC, Departamento de Física, FCT, Universidade Nova de Lisboa, 2829-516, \\ Campus de Caparica, Portugal \\ dm.garcia@campus.fct.unl.pt, ${ }^{*} \mathrm{dl} @$ fct.unl.pt, jla@campus.fct.unl.pt
}

\begin{abstract}
A novel solar laser uniformly pumped by six Fresnel lenses is proposed here. The incident solar radiation is firstly collected and concentrated by six $0.8 \mathrm{~m}$ diameter Fresnel lenses and then reflected by other six plane mirrors to a central focal zone, where a laser head is mounted. About $2.5 \mathrm{~kW}$ solar power with $3.5 \mathrm{~W} / \mathrm{mm}^{2}$ peak solar flux can be achieved in the focal zone. The laser head is composed of a fused silica six-sphere type secondary concentrator that further compresses the concentrated solar power from the six Fresnel lenses-plane mirrors to a core-doped YAG Nd ${ }^{3+}: \mathrm{YAG}$ ceramic disk. Optimum pumping parameters and solar laser output powers are found through ZEMAX non-sequential ray-tracing and LASCAD laser cavity analysis, respectively. The laser resonant cavity is formed by a PR $1064 \mathrm{~nm}$ output coupler and a HR $1064 \mathrm{~nm}$ plane reflector. An $8 \mathrm{~mm}$ diameter central hole is drilled through the six-sphere type concentrator to allow the extraction of laser power from the disk. Since only $16 \%$ of the useful solar power is absorbed by the Nd:YAG medium, for $950 \mathrm{~W} / \mathrm{m}^{2}$ of terrestrial solar irradiation, the effective solar pump power of $456 \mathrm{~W}$ is assumed in ray-tracing analysis. $72.2 \mathrm{~W}$ of multimode laser power is predicted for an $8 \mathrm{~mm}$ diameter gain medium embedded within a conical undoped YAG cladding, reaching the collection efficiency of $24.1 \mathrm{~W} / \mathrm{m}^{2}$. M $\mathrm{M}^{2}=16.6$ is numerically calculated, corresponding to the brightness figure of merit of $0.26 \mathrm{~W}$. A near uniform absorbed pump profile is achieved.
\end{abstract}

Keywords: Solar-pumped lasers, Nd:YAG, laser beam, beam profile, Fresnel lens, core-doped

\section{Introduction}

Solar-pumped lasers have gained an ever increasing importance in recent years [1]. Compared to electrically powered lasers, solar lasers are much simpler and reliable due to the complete elimination of the electrical power generation and power conditioning equipment. Nevertheless, additional focusing systems are usually required to collect solar radiation because natural sunlight does not provide power density sufficient enough to produce laser.

The first solid-state solar-pumped laser has been reported by Young in 1966 [2]. Since then, researchers have been exploiting parabolic mirrors and Fresnel lenses to attain enough concentrated solar radiation at focal point, and several pumping schemes have been proposed for enhancing solar laser output performance [3-7]. The progress with Fresnel lenses and chromium co-doped Cr:Nd:YAG ceramic laser medium [8] has revitalized solar laser researches. $19.3 \mathrm{~W} / \mathrm{m}^{2}$ collection efficiency has been reported in 2011 [5] by utilizing an economical Fresnel lens and the most widely used $\mathrm{Nd}$ :YAG single-crystal rod. Most recently, record-high Nd:YAG solar laser collection efficiency of $30.0 \mathrm{~W} / \mathrm{m}^{2}$ has been achieved [9] through a Fresnel lens scheme. Despite the strong desire to achieve high collection efficiency, much more attentions should also be paid to the solar laser beam quality in order to attain tight focusing, which is very important for most laser applications.

The thin-disk laser concept, initially developed for diode-pumped laser systems [10,11], is one of the most suitable approaches when high power, high efficiency and good beam quality are required simultaneously. Due to small volumeto-surface-area ratio, the gain medium can be cooled very efficiently. The direction of the heat flow is hence mainly parallel to the laser cavity axis, which in combination with short optical path length through the active medium, results in a reduction of the thermal lensing effect and thermally induced aberrations by orders of magnitude compared to typical high-power rod lasers. Typical thin disk laser systems use a nearly end-pumped geometry, however, the small absorption

RIA100 - 682 V. 1 (p.1 of 7) / Color: No / Format: A4 / Date: 5/15/2013 8:53:30 AM

SPIE USE: DB Check, Prod Check, Notes: 
Return to the Manage Active Submissions page at http://spie.org/app/submissions/tasks.aspx and approve or disapprove this submission. Your manuscript will not be published without this approval. Please contact author_help@spie.org with any questions or concerns.

distance inside the active medium yields poor absorption. A solution to this problem has been proposed by Dascalu et. al. [12], consisting of a laser disc diffusion bonded to an un-doped YAG cap of larger dimensions. The pump beams are delivered across three windows cut obliquely on the pumping surface of the un-doped YAG medium. The incoming light rays then propagates inside the un-doped cap and the gain medium through total internal reflection. This configuration allows the multi-pass pumping through the gain medium, providing a way to concentrate the pump light uniformly into a smaller diameter disk. The integration of this approach to solar lasers through optical fiber pumping has been proposed [13] for the alleviation of the thermal management problems that has plagued the present-day solar-pumped lasers. Nevertheless, this solar pumping scheme requires a large number of optical fibers and small primary collectors, resulting in the collection efficiency of $5.9 \mathrm{~W} / \mathrm{m}^{2}$.

To attain both high solar laser collection efficiency and high quality solar laser beam, a new solar pumping approach is here proposed for efficient side-pumping of the core-doped YAG Nd:YAG disk. Core-doped ceramic mediums are laser active in the Nd-doped core only, and bonded with the same host material either un-doped or doped with a different element that effectively absorbs light at the signal wavelength. Since the medium cross-section is widened by the cladding, wider Gaussian intensity distributions can be accommodated in the laser active region without truncating its wings. The negative impact of diffraction on the laser beam quality can hence be reduced [14].

Solar energy collection and concentration is achieved through the combination of six Fresnel lenses - plane mirrors. The concentrated solar radiation is then focused to the lateral face of the disk through the six fused silica sphere type concentrator. Optimum pumping conditions and solar laser beam parameters are found through ZEMAX and LASCAD numerical analysis, respectively, for different core-doped Nd:YAG ceramic disks with different cladding slope angles. The production of $72.2 \mathrm{~W}$ multimode laser power is expected with an $8 \mathrm{~mm}$ diameter, $6 \mathrm{~mm}$ thickness Nd:YAG gain media embedded within a $34^{\circ}$ slope angle YAG cladding. High solar laser collection efficiency of $24.1 \mathrm{~W} / \mathrm{m}^{2}$ is hence achieved. Beam quality factor $\mathrm{M}^{2}=16.6$ is numerically attained in this case, resulting in the brightness figure of merit of $0.26 \mathrm{~W}$, which approaches the record value for Nd:YAG solar laser.

\section{Solar pumping approach for core-doped Nd:YAG disk laser}

\subsection{Solar energy collection and concentration through the six Fresnel lenses-plane mirrors system}

The solar-pumped disk laser scheme in Fig 1 (a) is formed by a combination of 6 pairs of circular Fresnel lenses and plane mirrors for edge-pumping of the core-doped ceramic Nd:YAG disk. Solar tracking can be achieved by mounting the whole laser system onto a two axis-heliostat that follows the Sun continuously in direct tracking mode. The incoming solar radiation is firstly collected and concentrated by the Fresnel lenses. They are evenly distributed along an $800 \mathrm{~mm}$ radius virtual circumference, which center is located $370 \mathrm{~mm}$ below the center of the laser head, as shown in Fig. 2. To both reflect and concentrate the solar rays onto the laser head, each plane mirror is then placed $710 \mathrm{~mm}$ below the corresponding Fresnel lens, with a $74^{\circ}$ inclination in relation to their common optical axis.
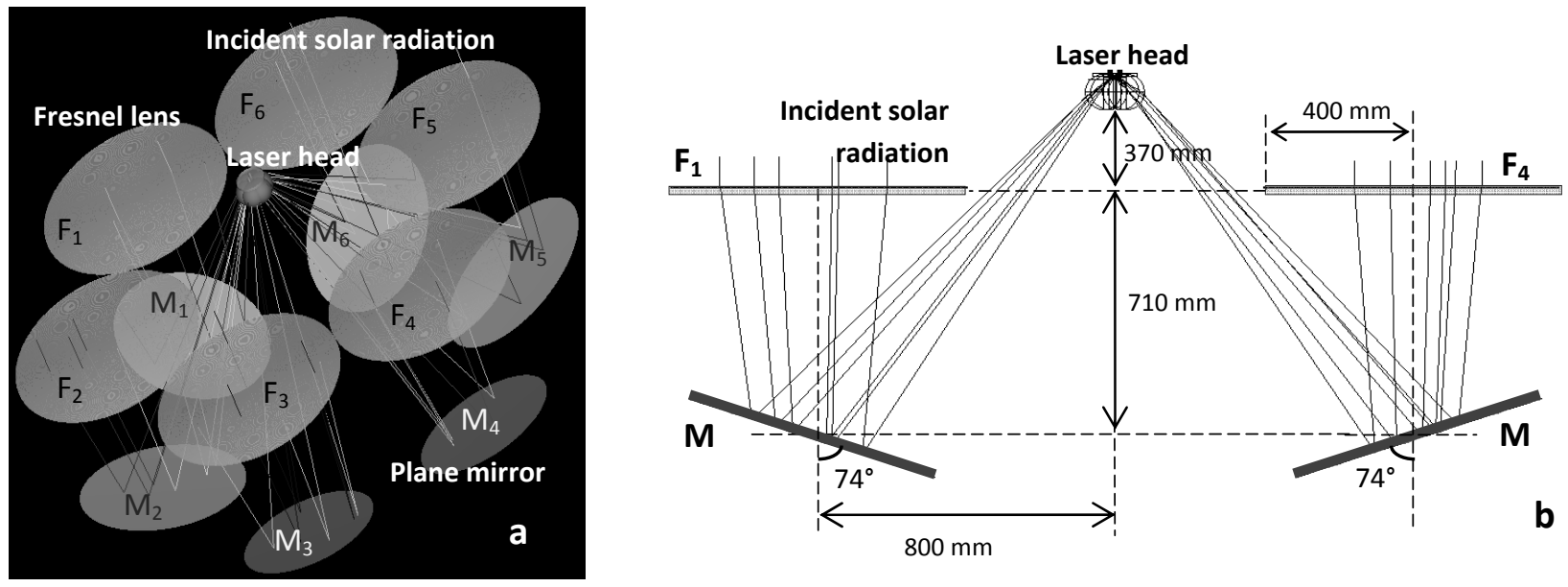

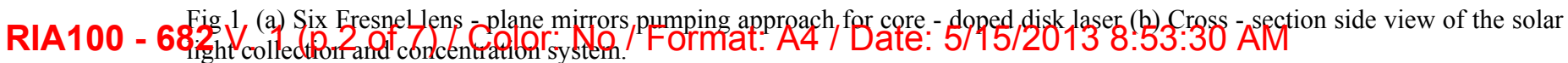


Return to the Manage Active Submissions page at http://spie.org/app/submissions/tasks.aspx and approve or disapprove this submission. Your manuscript will not be published without this approval. Please contact author_help@spie.org with any questions or concerns.

Each Fresnel lenses has $0.4 \mathrm{~m}$ radius and $2.2 \mathrm{~m}$ focal length. It is made of Polymethyl Methacrylate (PMMA) material, which is transparent at visible and near infrared wavelengths, but absorbs the infrared radiation beyond $2200 \mathrm{~nm}$ and cut undesirable UV solar radiation below $350 \mathrm{~nm}$. An averaged transmission efficiency of $90 \%$ is numerically calculated for each Fresnel lens. Considering $98 \%$ reflectivity for each plane mirror and the terrestrial solar irradiation of $950 \mathrm{~W} / \mathrm{m}^{2}$, about $2.5 \mathrm{~kW}$ solar power with $3.5 \mathrm{~W} / \mathrm{mm}^{2}$ peak flux can be achieved in the focal zone. This value can be improved to 9 $\mathrm{W} / \mathrm{mm}^{2}$ with the fused silica six spheres type secondary concentrator, in Fig. 2 (a), which minimizes the focal spot and compresses the concentrated solar radiation from the six Fresnel lenses-plane mirrors to the laser disk.

\subsection{The core-doped Nd:YAG laser head}

As shown Fig. 2 (b), the secondary concentrator is formed by the union of six fused silica spheres, with $50 \mathrm{~mm}$ diameter. The formation has a six-fold symmetry, in which the center of each sphere is placed $31 \mathrm{~mm}$ away from the center of the concentrator. This geometry allows the more efficient compression of the pump light from each Fresnel lens-plane mirror.
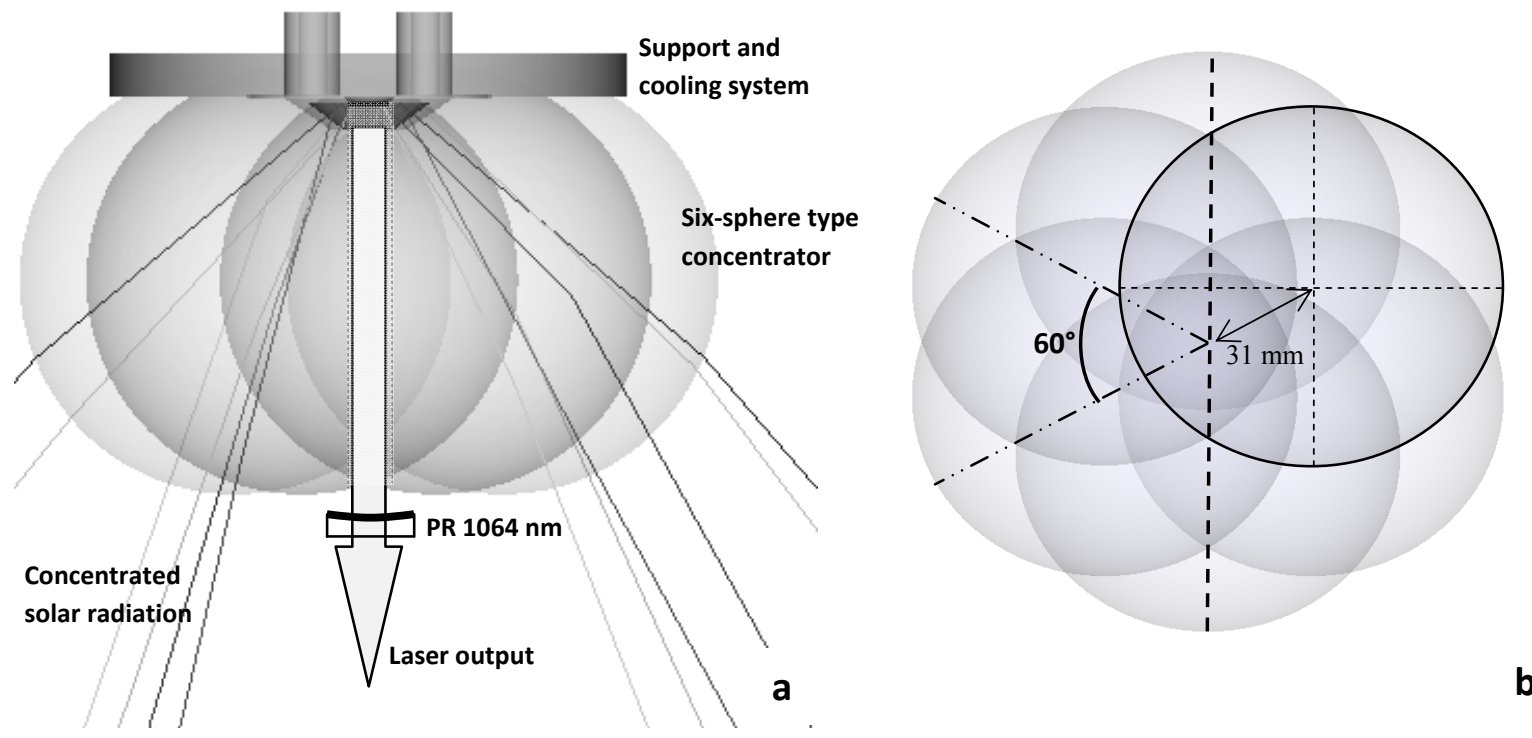

Fig 2. (a) The fused silica six-sphere type second stage concentrator. (b) Detailed view of the union of six-spheres.

Fused silica is an ideal optical material for Nd:YAG laser pumping since it is transparent over the Nd:YAG absorption spectrum. It has a high softening point and is resistant to scratching and thermal shock. The fused silica material is also important in preventing the possible heating of ultra-high solar flux at the focus to the mechanical structure of the laser head. High optical quality ( $99.999 \%$ ) concentrators can be manufactured by optical machining and polishing. A conical hole is drilled in the concentrator for the installation of the conical core-doped laser disk, as shown in Fig. 3. Both upper and lateral faces of the disk are directly cooled by water, whereas the lower surface is fixed to the concentrator with optical glue. The cooled upper surface of the gain medium is HR $1064 \mathrm{~nm}$ coated, whereas the lower surface is AR $1064 \mathrm{~nm}$ coated. A central hole is drilled along the six-sphere type concentrator to allow the extraction of laser power. The laser resonant cavity is hence formed by both the HR $1064 \mathrm{~nm}$ coating and the output coupler, as shown in both Fig. 2 (a) and Fig. 3. 
Return to the Manage Active Submissions page at http://spie.org/app/submissions/tasks.aspx and approve or disapprove this submission. Your manuscript will not be published without this approval. Please contact author_help@spie.org with any questions or concerns.

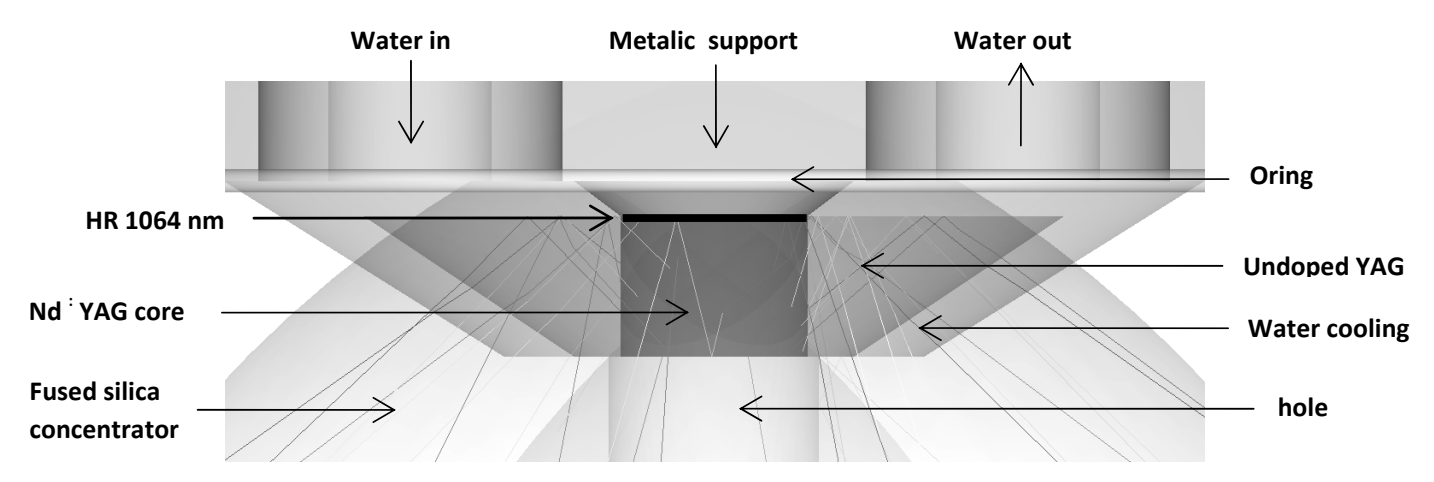

Fig 3.Cooling system and mechanical support of the core-doped Nd:YAG laser head.

With this new configuration, the concentrated pump light from each Fresnel lens-plane mirror is delivered across the oblique lateral surface of the un-doped YAG element, $60^{\circ}$ apart from each other, as illustrated in Fig.4 (b). The pumping rays then propagate inside the core-doped disk through total internal reflection. This enables a multi-pass pumping inside the disk, improving both the absorbed pump power and absorbed pumped distribution within the smaller diameter gain medium, providing a way to concentrate the pump beam uniformly into a smaller diameter medium.

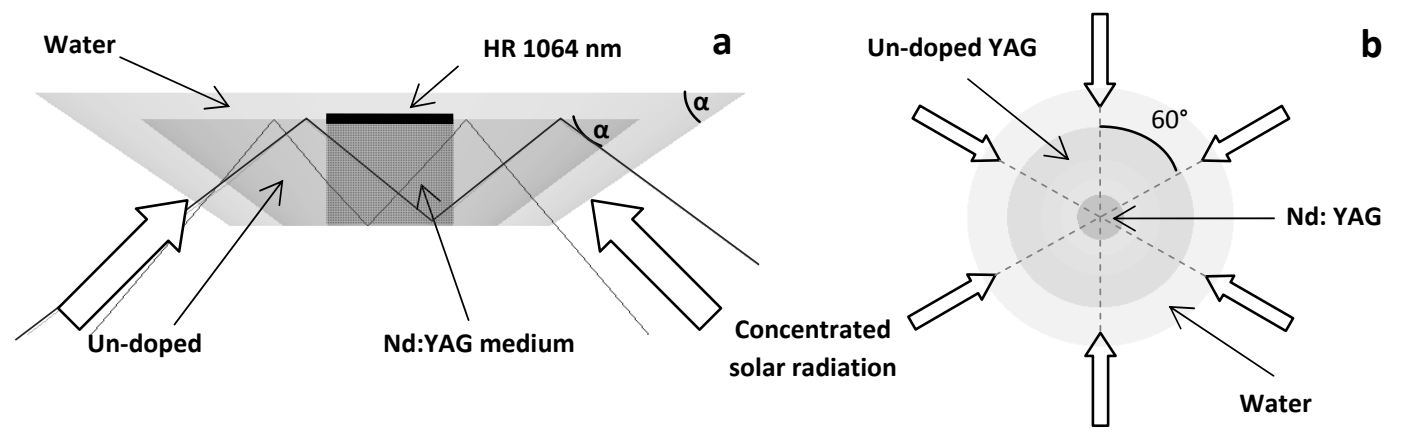

Fig 4. Pump light concentration and propagation through the conical core-doped YAG Nd:YAG laser disk:

(a) Cross-sectional side view and (b) top view.

\section{Numerical analysis of solar laser performances with core-doped Nd:YAG disks}

Optimum pumping parameters for the whole optical system are found through ZEMAX non-sequential ray-tracing with merit function optimization. The standard solar spectrum for one-and-a-half air mass (AM1.5) [15] is used as the reference data for consulting the spectral irradiance $\left(\mathrm{W} / \mathrm{m}^{2} / \mathrm{nm}\right)$ at each wavelength. Taking into account the $16 \%$ overlap between the solar spectrum and the Nd:YAG medium absorption spectrum [16], the effective solar pump power of $456 \mathrm{~W}$ is assumed in ZEMAX non-sequential ray-tracing analysis. The solar half-angle divergence of $0.27^{\circ}$ is also considered. The absorption spectrum of all materials is included in ZEMAX numerical data to account for absorption losses.

Nd:YAG is the most-widely used solid-state laser material and is a promising candidate in high-power thin-disk lasers. It has been demonstrated as the best material under solar pumping because of its superior characteristic on thermal conductivity, high quantum efficiency and mechanical strength compared to other host materials [2-5, 7]. For $1.1 \%$ $\mathrm{Nd}$ :YAG laser medium, 22 absorption peaks are defined in ZEMAX numerical data [3-5]. All the peak wavelengths and their respective absorption coefficients are added to the glass catalogue for Nd:YAG material in ZEMAX software. Solar irradiance values for the above-mentioned 22 peak absorption wavelengths could be consulted from the standard solar spectra for AM1.5 and saved as source wavelength data. In ray-tracing, the laser disk is divided into a total of 18000 zones. The path length in each zone is found. With this value and the effective absorption coefficient of $1.1 \% \mathrm{Nd}$ :YAG 
Return to the Manage Active Submissions page at http://spie.org/app/submissions/tasks.aspx and approve or disapprove this submission. Your manuscript will not be published without this approval. Please contact author_help@spie.org with any questions or concerns.

material, the absorbed power within the laser medium can be calculated by summing up the absorbed pump radiation of all zones. The absorbed pump flux data from the ZEMAX analysis is then processed by LASCAD software to study the laser performance. All the technical parameters and data used in both ZEMAX and LASCAD numerical analysis have already been confirmed by our previous experimental results [3-5].

In LASCAD analysis the optical resonator is comprised of two opposing parallel mirrors at right angles to the axis of the laser disk. The amount of feedback is determined by the reflectivity of the mirrors. One end mirror is high reflection coated (HR, $99.98 \%$ ) and corresponds to the HR-coated surface of the disk. The output coupler is partial reflection coated (PR) with reflectivity variable between 90 - $99 \%$, according to different disk diameters.

For correct parameterization of the laser system, the laser output powers of core-doped disks with different gain medium diameters $\left(D_{\mathrm{Nd}: \mathrm{YAG}}\right)$ are studied as function of the cladding slope angle $(\alpha)$. The core-doped disk thickness is maintained at $6 \mathrm{~mm}$ for all cases. A $400 \mathrm{~mm}$ length resonator is chosen in LASCAD software. The radius of curvature of the output coupler $\mathrm{RoC}=5 \mathrm{~m}$ is assumed in the analysis.

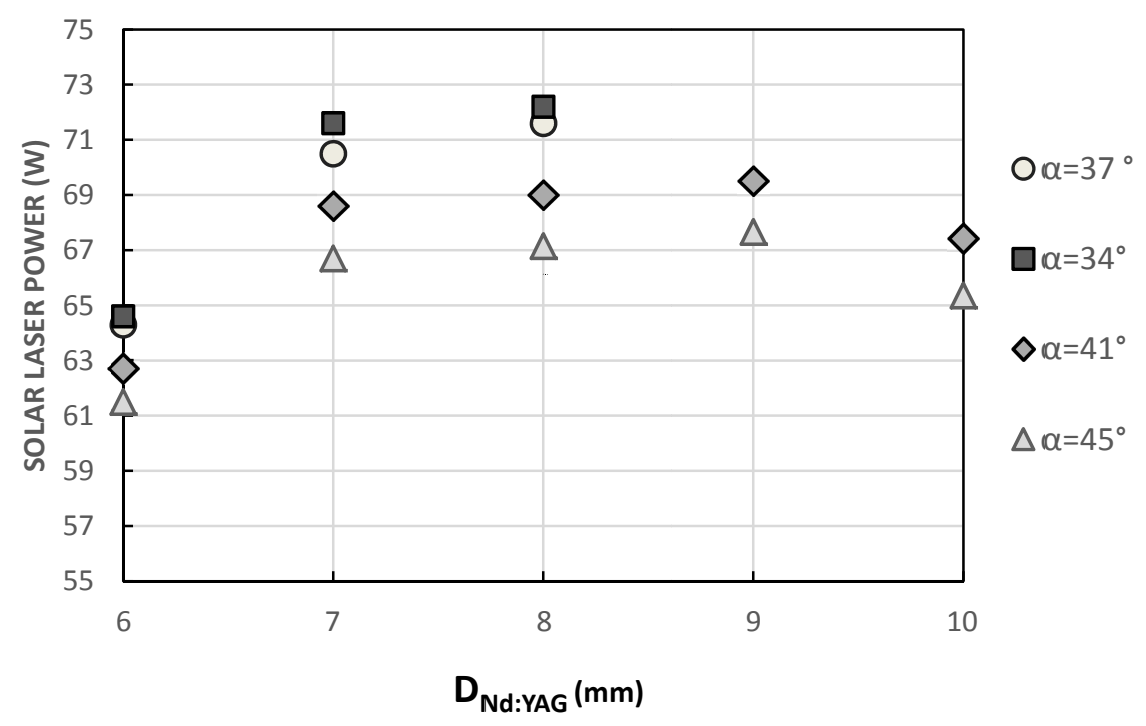

Fig 5. Calculated multimode solar laser power output for different doped core diameters $\left(\mathrm{D}_{\mathrm{Nd}: \mathrm{YAG}}\right)$ as the function of the cladding slope angle $(\alpha)$.

The cladding slope angle of $34^{\circ}$ allows more absorbed pumped power within the gain medium, resulting in higher multimode laser power compared to other angles, as given in Fig. 5. The maximum laser output power of $72.2 \mathrm{~W}$ is numerically achieved with the $\mathrm{D}_{\mathrm{Nd}: \mathrm{YAG}}=8 \mathrm{~mm}$, resulting in high collection efficiency of $24.1 \mathrm{~W} / \mathrm{m}^{2} . \mathrm{M}^{2}=16.6$ are numerically calculated in these case, resulting in the brightness figure of merit of $0.26 \mathrm{~W}$, which approaches the record value for Nd:YAG solar laser. Due to mechanics confinement, the core-doped disks with $\mathrm{D}_{\mathrm{Nd}: Y A G}$ higher than $8 \mathrm{~mm}$ cannot be numerically calculated for the slope angles minor than $40^{\circ}$. In these cases, $\alpha=41^{\circ}$ offers the best option to attain maximum laser power.

The grey-scale absorbed pump flux distribution within the central cross-sections of the $\mathrm{D}_{\mathrm{Nd}: \mathrm{YAG}}=8 \mathrm{~mm}$ core-doped disk with $34^{\circ}$ cladding slope angle is shown in Fig. 6. Black color means near maximum pump absorption for these plots, whereas white means little or no absorption. A near uniform profile is achieved, which contributes largely to the reduction of the laser beam divergence and thermal loading problems. 
Return to the Manage Active Submissions page at http://spie.org/app/submissions/tasks.aspx and approve or disapprove this submission. Your manuscript will not be published without this approval. Please contact author_help@spie.org with any questions or concerns.

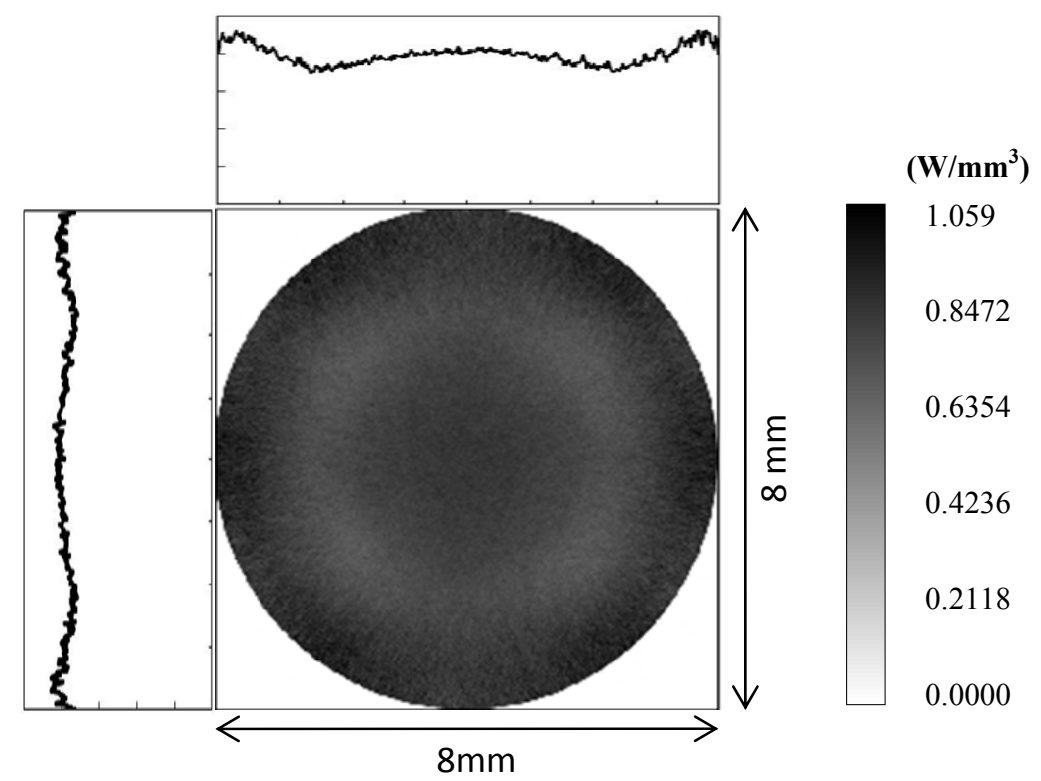

Fig 5. Absorbed pump flux distribution along the central cross-section of the $8 \mathrm{~mm}$ diameter Nd:YAG core.

\section{Conclusions}

Based on thin-disk laser technology and on our experience in solar-pumped solid-state lasers, an alternative six Fresnel lenses - plane mirrors approach is here proposed for the efficient side-pumping of the conical core-doped YAG Nd:YAG ceramic disk. In addition to the high concentration capacity of the fused silica six sphere type secondary concentrator, $72.2 \mathrm{~W}$ solar laser power can be achieved by the $34^{\circ}$ slope angle core-doped disk with $8 \mathrm{~mm}$ diameter doped core, corresponding to high collection efficiency of $24.1 \mathrm{~W} / \mathrm{m}^{2}$. A brightness figure of merit of $0.26 \mathrm{~W}$ is expected in this case. A near uniform absorbed pump flux distribution is achieved. The proposed solar pumping scheme presents therefore a good compromise between laser power and beam quality.

\section{Acknowledgments}

These research projects (PTDC/FIS/103599/2008 and PTDC/FIS/122420/2010) were funded by the Science and Technology Foundation of Portuguese Ministry of Science, Technology and Higher Education (FCT-MCTES).

\section{References}

[1] Graham-Rowe, D., "Solar-powered laser," Nat. Photonics 4, 64-65 (2010).

[2] Young, C. G., “A sun pumped cw one-watt laser,” Appl. Opt. 5(6), 993-997, (1966).

[3] Weksler, M. and Shwartz, J., "Solar-pumped solid-state lasers," IEEE J. Quantum Electron. 24(6), 12221228 (1988).

[4] Lando, M., Kagan, J., Linyekin, B. and Dobrusin, V., "A solar pumped Nd:YAG laser in the high collection efficiency regime," Opt. Commun. 222(1-6), 371-381 (2003).

[5] Liang, D. and Almeida, J., "Highly efficient solar pumped Nd:YAG laser," Opt. Express 19(27), 26399-26405 (2011). 
Return to the Manage Active Submissions page at http://spie.org/app/submissions/tasks.aspx and approve or disapprove this submission. Your manuscript will not be published without this approval. Please contact author_help@spie.org with any questions or concerns.

[6] Almeida, J., Liang, D. and Guillot, E., "Improvement in solar-pumped Nd:YAG laser beam brightness," Opt. Laser Technol. 44(7), 2115-2119 (2012).

[7] Almeida, J., Liang, D., Guillot, E. and Abdel-Hadi, Y.,"A $40 \mathrm{~W}$ cw Nd:YAG solar laser pumped through a heliostat : a parabolic mirror system," Laser Phys. 23 (2013) 065801.

[8] Yabe, T., Ohkubo, T., Uchida, S., Nakatsuka, M., Funatsu, T., Mabuti, A., Oyama, A., Nakagawa,Y., Oishi, T., Daito, K., Behgol, B., Nakayama,Y., Yoshida, M., Motokoshi, S., Sato, Y. and Baasandash, C., "High efficiency and economical solar energy pumped laser with Fresnel lens and chromium co-doped laser medium," Appl. Phys Lett. 90(26), 261120-3 (2007).

[9] Dinh, T. H., Ohkubo, T., Yabe, T. and Kuboyama, H., "120 watt continuous wave solar-pumped laser with a liquid light-guide lens and a Nd:YAG rod," Opt. Lett. 37(13), 2670-2672 (2012).

[10] Giesen, A., Hügel H., Voss A., Wittig, K., Brauch. U. and Opower. H., "Scalable concept for diode-pumped highpower solid-state lasers," Appl. Phys. B 58(5), 365-372 (1994).

[11] Giesen, A. and Speiser, J., "Fifteen years of work on thin-disk lasers: results and scaling laws," IEEE J. Sel. Top. Quantum Electron.13 (3), 598-609 (2007).

[12] Dascalu T. and Taira T., "Highly efficient configuration for microchip solid-state laser," Opt. Express 4(2), 670677 (2006).

[13] Couto, B. and Liang, D., "Optical fiber pumping of solar Nd:YAG disc laser," Proc. SPIE 8001, 80014D (2011).

[14] Sträßer, A., Ostermeyer, M., "Improving the brightness of side pumped power amplifiers by using core-doped ceramic rods," Opt. Express 14 (15), 6687-6693 (2006).

[15] ASTM Standard G173 (2012).

[16] Zhao, B., Zhao, C., He, J. and Yang, S.,"The Study of Active Medium for Solar-Pumped Solid-State Lasers," Acta Opt. Sin. 2007 (10), 1-9 (2006). 